\title{
GSDMD-dependent neutrophil extracellular traps formation contributes to fibroblast-like synoviocyte activation in rheumatoid arthritis
}

\section{Kaifeng Zhou}

Fudan University

Chong Bian

Fudan University

Huijie Gu

Fudan University

\section{Xiangyang Cheng}

Fudan University

\section{Zhongyue Huang}

Fudan University

\section{Guangnan Chen}

Fudan University

Jun Xu

Department of orthopaedics, Minhang Hospital

Xiaofan Yin ( $\sim 18918169029 @ 189 . c n$ )

\section{Research}

Keywords: Gasdermin D, neutrophil, rheumatoid arthritis, fibroblast-like synoviocyte

Posted Date: April 13th, 2021

DOl: https://doi.org/10.21203/rs.3.rs-401936/v1

License: (c) (i) This work is licensed under a Creative Commons Attribution 4.0 International License.

Read Full License 


\section{Abstract \\ Objective}

The activation of NLRP3 inflammasome is critical for rheumatoid arthritis (RA), however, the role of gasdermin D (GSDMD), a newly identified pyroptosis executioner downstream of NLRP3/ASC/Caspase-1, in RA has not been well defined.

\section{Methods}

Genetically susceptible mice (DBA/1J) are immunized with a type II bovine collagen emulsion in complete Freund's adjuvant (CFA). Peripheral blood neutrophils were isolated from healthy volunteers and RA patients; cells were treated with serum from RA patients. The activation of GSDMD was analyzed by confocal and western blot. Proinflammatory cytokines in joint of mice were detected by qPCR and ELISA.

\section{Results}

The expression of cleaved GSDMD-N terminal in peripheral blood neutrophils from RA patients and the expression of GSDMD-N was positively correlated with the level of IL-1 $\beta$ and IL-18 from RA patients. GSDMD was required for the pathogenesis of RA, and GSDMD inhibition disulfiram significantly suppressed proinflammatory cytokines production and joint damage, leading to reduced arthritis severity score and loss of cartilage in collagen-induced arthritis (CIA) mouse model. Mechanistically, the activation of GSDMD significantly promotes neutrophil extracellular traps (NETs), which are highly immunogenic and decorated with HMGB1 and MMPs. Furthermore, GSDMD-dependent NETs formation facilitated fibroblast-like synoviocytes (FLS) activation and proliferation, driving cartilage and bone destruction, and promoting disease activity in RA.

\section{Conclusions}

GSDMD-dependent NETs formation promotes FLS activation during RA pathogenesis.

\section{Introduction}

Rheumatoid arthritis (RA) is a chronic, progressive and invasive disease that cause the loss of joint function and major disabilities [1]. Joint inflammation and synovial hyperplasia are characterized as leading cause to joint destruction. At the site of inflammation, inflammatory cells, such as neutrophils, macrophages, $T$ and B cells are recruited and further activated [2]. These cells also activate joint cells, including fibroblast-like synoviocytes (FLS), chondrocytes, and osteoclasts, which increase inflammation and induce cartilage degradation and bone erosion [3]. However, the precise mechanism between immune cells and resident joint cells are poorly studied. 
Neutrophils are abundant in both synovial tissue and fluid, and play an important role in initial pathogenesis in RA [4]. Recent studies indicated that neutrophils from RA synovial and peripheral blood show a significant enhanced capacity to form neutrophil extracellular traps (NETs) [5, 6]. During NET formation, neutrophils extrude a meshwork of nuclear material coupled to cytoplasmic and granular proteins including elastase, cathepsin G and MMP9. Peptidylarginine deiminase-4 (PAD4), a myeloidspecific PAD involved in citrullination, which have been characterized as important source of autoantigens in RA [5]. Recently, several studies have suggested that the activation of Gasdermin D (GSDMD) is required in the generation of NETs [7, 8]. However, whether GSDMD activation is involved in neutrophil death and contribute to disease activity in RA has not been elucidated.

GSDMD is recently identified pore-forming protein, which mediates pyroptosis $[9,10]$. Proinflammatory caspase (e.g., Caspase-1, Caspase-4, Caspase-5, and Caspase-11) can cleave GSDMD at D276/G277 sites, thereby releasing the autoinhibition by C-terminal GSDMD domain on the N-terminal GSDMD domain. The cleaved GSDMD-N then translocates to the plasma membrane, where they oligomerize to form membrane pores, leading to cell lysis and secretion of IL-1 $\beta$ and IL-18 [11, 12]. Recent studies demonstrate that GSDMD plays a critical role in autoimmune disease, such as familial Mediterranean fever or experimental autoimmune encephalomyelitis $[13,14]$. Interestingly, a recent study reported that CD14 ${ }^{+}$monocytes of RA patients undergo increased levels of GSDMD-N. However, to data there have been no reports on whether GSDMD can be a therapeutic target in RA.

Fibroblast-like synoviocytes (FLS), also known as synovial fibroblasts or type B synoviocytes, are major effectors in cartilage damage and participate in synovial inflammation in the rheumatoid joint [15]. The main role of fibroblast is to provide structural support, lubrication to allow low friction movements of the articular joints and nutrients to the vascular cartilage [16]. Recent evidence demonstrates that FLS are activated by NETs, leading to up-regulation of inflammatory cytokine and adhesion molecule synthesis [17]. However, the mechanisms by how NETs activate FLS remain to be fully characterized.

We now provide the first insight into the physiological function of GSDMD in RA pathogenesis and describe the regulatory mechanism of GSDMD-mediated pyroptosis in the development of RA. We discover that neutrophil GSDMD activation is crucial for proinflammatory cytokines production and NETs, which facilities FLS activation in RA.

\section{Materials And Methods}

Materials Chemicals utilized for molecular and cellular biology and buffer preparation were purchased from Sangon Biotech (Shanghai). Cell culture medium including Dulbecco's Modified Eagle's Medium, and supplements including penicillin and streptomycin (Cat\#: 15140-122, Gibco, Waltham, MA, USA) were purchased from Life Science Technologies (Rockville, MD, USA). Antibodies to GSDMD (ab219800), Fibronectin (ab2413), Collagen I (ab34710), a-SMA (55135-1-AP), Histone-cit H3 (ab219407) was obtained from Abcam. Anti-casapse-11 (17D9, C1354) was from Sigma-Aldrich. Anti-caspase-1(AC-20B00420-C100) was from Adipogen. $\beta$-actin (3700S) were purchased from Cell Signaling Technology. Anti- 
Ly6G (127626) was purchased from Biolegend. Fetal bovine serum (Cat\#: 26140-079, Gibco) was purchased from Life Science Technologies and heat inactivated before utilization.

Animals $G s d \mathrm{md}^{\text {t- }}$ mice were obtained from the model animal research center of Nanjing University (Jiangsu, China). Casp 1\%-Casp $11^{\%}$ mice were kindly provided by Dr. Feng Shao (National Institute of Biological Sciences, Beijing, China). All animal experiments were performed according to the Criteria of the Medical Laboratory Animal Administrative Committee of Shanghai and the Guide for Care and Use of Laboratory Animals of Secondary Military Medical University.

Isolation and stimulation of human peripheral neutrophils Neutrophil isolation was completed according to the method of human peripheral blood granulocytes [18] as described briefly: Blood was layered on Histopaque 1119 (Sigma-Aldrich) and centrifuged for $20 \mathrm{~min}$ at $800 \mathrm{x} \mathrm{g}$. The granulocyte-rich layer below the interphase was collected and further fractionated on a discontinuous Percoll gradient consisting of layers with densities of $1105 \mathrm{~g} / \mathrm{ml}(85 \%), 1100 \mathrm{~g} / \mathrm{ml}(80 \%), 1093 \mathrm{~g} / \mathrm{ml}(75 \%), 1087 \mathrm{~g} / \mathrm{ml}$ (70\%), and 1081 $\mathrm{g} / \mathrm{ml}(65 \%)$. After centrifugation for $20 \mathrm{~min}$ at $800 \times \mathrm{g}$, the interface between the $80 \%$ and $85 \%$ Percoll layers was collected and washed twice in RPMI 1640 medium. All procedures were conducted at room temperature. The preparations contained $99 \%$ granulocytes, of which $95 \%$ were neutrophils and $1-4 \%$ were eosinophils, as determined by Giemsa staining after cytocentrifuge preparation. Serum was collected from peripheral blood of RA patients, and neutrophils was treated with RA serum at $10 \%$. For human studies, written informed consent from participants and parents or legal guardians where applicable was received under protocols approved by the ethics committee of Minhang Hospital.

Conduction of CIA mouse model DBA/J1 mice (20 males, 6 weeks old) purchased from Joint Ventures Sipper BK Experimental Animal Co. (Shanghai, China) were acclimated for 1 week with on a 12-h dark/light cycle and allowed food and water ad libitum. To induce arthritis, DBA/1 J mice were intradermally injected at the base of the tail with $100 \mu \mathrm{g}$ of type II bovine collagen in complete Freund's adjuvant (1:1 w/v; Chondrex, Redmond, WA, USA) and boosted intradermally 14 days later. Arthritic score measurements were performed as follows: $0=$ no joint swelling; 1 = slight edema and erythema limited to the foot or ankle; 2 = slight edema and erythema from the ankle to the tarsal bone; 3 = moderate edema and erythema from the ankle to the tarsal bone; and $4=$ edema and erythema extending from the ankle to the entire leg, with severe swelling of the wrist or ankle. The final arthritis score was calculated as the sum of scores from all four legs, which were assessed by three independent observers with no knowledge of the experimental groups. The mice were randomly divided two groups (vehicle and kaempferol injection groups).

Flow cytometry analysis For surface staining, $100 \mathrm{ul}$ blood were harvested, and blocked with Fc blocker (Biolegend) for $30 \mathrm{~min}$ at $4^{\circ} \mathrm{C}$. The cells were resuspended in $50 \mu \mathrm{l}$ FACS buffer ( $1 \times$ PBS with $0.5 \% \mathrm{FBS}, 2$ mM EDTA) with diluted GSDMD antibody and incubated for $30 \mathrm{~min}$ at $4^{\circ} \mathrm{C}$. The cells were stained with anti-rabbit FITC (ab7086, abcam, 1:2000) for $30 \mathrm{~min}$ at $4^{\circ} \mathrm{C}$. The cells were washed twice by FACS buffer and analyzed by flow cytometry. For the intracellular GSDMD staining, an additional staining step was performed using Cytofix/Cytoperm Fixation/Permeabilization Solution Kit (BD Biosciences) according to 
the manufacturer's instructions. Data were acquired on a BD Fortessa X20 (BD Biosciences) and analyzed using FlowJo software (Tree Star, Inc.).

Isolation of mouse bone marrow neutrophils Femurs and tibias were collected and bone marrow was flushed into a $50 \mathrm{ml}$ conical tube with HBSS. Following ACK lysis, neutrophils were purified through a $62.5 \%$ percoll gradient. The purity of isolated cells has reached over $90 \%$ as determined by flow cytometry. Neutrophils were treated with $10 \%$ serum from CIA mice.

Isolation of FLS and dermal fibroblasts Human OA and RA FLS were obtained, as previously described (18). Simplify, FLS were obtained by collagenase (Roche) digestion of human synovial tissue obtained at arthroplasty or synovectomy from RA or OA joints. Cells were maintained in CMRL medium (Invitrogen Life Technologies) and used after passage 4 from primary cultures. The cells were cultured in DMEM supplemented with $10 \%$ fetal bovine serum (FBS; Invitrogen), $2 \mathrm{mM}$ glutamine, penicillin $(100 \mathrm{U} / \mathrm{ml})$, and streptomycin $(100 \mathrm{~g} / \mathrm{ml})$ and grown in a humidified incubator with $5 \% \mathrm{CO} 2$ at $37^{\circ} \mathrm{C}$.

Cell culture The primary FLS cells obtained from Changhai Hospital. Cells were treated with $2 \mu \mathrm{g} / \mathrm{ml}$ of puromycin for 3 days to eliminate the non-infected cells. Synoviocytes were isolated by enzymatic digestion of synovial tissue specimens. The tissue samples were minced into $2-3 \mathrm{~mm}$ pieces and treated for $4 \mathrm{~h}$ with $4 \mathrm{mg} / \mathrm{ml}$ type II collagenase (Worthington, Freehold, $\mathrm{NJ}$ ) in DMEM at $37^{\circ} \mathrm{C}$ in $5 \% \mathrm{CO} 2$. Dissociated cells were centrifuged at $500 \mathrm{~g}$, resuspended in DMEM supplemented with $10 \%$ fetal calf serum, $2 \mathrm{mM}$ L-glutamine, $100 \mathrm{unit} / \mathrm{ml}$ penicillin, and $100 \mathrm{ng} / \mathrm{ml}$ streptomycin incubated overnight. The non-adherent cells were then removed, and the adherent cells were cultivated in DMEM supplemented with $10 \%$ fetal calf serum.

Real-time quantitative PCR Extraction of RNA and operation of real-time quantitative PCR were performed by Fastagen RNA extraction and TOYOBO RT-PCR kits according to the protocols. Sequences of the primers for targeting of IL-1 $\beta$, IL-6, IL-8 and TNF-a expression are in follow. II-1 $\beta$ : Forward: 5'TGGACCTTCCAGGATGAGGACA-3', 5'-GTTCATCTCGGAGCCTGTAGTG-3'; IL-6: 5'AAGCCAGAGCTGTGCAGATGAGTA-3', Reverse: 5'-CTTGGTCACCGACGTCCTGT-3'; IL-8: 5'AAGGCTGGTCCATGCTCC-3', Reverse: 5'-TGCTATCACTTCCTTTCTGTTGC-3'; TNF-a: 5'GAAAGCATGATCCGGGACGTG-3', Reverse: 5'-GATGGCAGAGAGGAGGTTGAC-3'; MMP1: 5'CAGAGATGAAGTCCGGTTTTTC-3', Reverse: 5'- GGGGTATCCGTGTAGCACAT-3'; MMP3: 5'CAAAACATATTTCTTTGTAGAGGACAA-3', Reverse: 5'- TTCAGCTATTTGCTTGGGAA-3'; GAPDH: 5'TGGAAGGACTCATGACCACA-3', Reverse: 5'-AGGGGTCTACATGGCAACTG-3'. Data was normalized by the level of $\beta$-actin expression in each sample.

Enzyme-linked immunosorbent assay (ELISA) IL-1 $\beta$, IL-6, IL-8, IL-18 and TNF- $\alpha$ in the supernatants were measured by ELISA kits (R\&D Systems) according to the protocols of the manufacturer. MMP1 and MMP3 in the supernatants were measured by ELISA kits (eBiosciences) according to the protocols of the manufacturer. 
Western blotting Each equal amount of protein was resolved by $10 \%$ sodium dodecyl sulfatepolyacrylamide gel electrophoresis (SDS-PAGE) and transferred onto polyvinylidene difluoride (PVDF, Merck Millipore Ltd, Burlington, MA, USA) membranes. The membranes were then incubated in a blocking buffer containing $5 \%$ non-fat milk/1 $\times$ TBST, and probed with specific indicated antibodies, respectively. The proteins were visualized by an enhanced chemiluminescence detection system.

Immunofluorescence of NETs Neutrophils were seeded onto poly-d-lysine-coated coverslips (Corning, NY, USA) and NETs were induced according to the abovementioned protocol. Fixed neutrophils were blocked with $1 \%$ BSA and $5 \%$ normal goat serum in PBS and stained with polyclonal rabbit neutrophil elastase in PBS and incubated for $12 \mathrm{~h}$ at $4{ }^{\circ} \mathrm{C}$. Then, neutrophils were washed and incubated with a $1 / 500$ dilution secondary antibodies (Life Technologies) and Sytox Green for $2 \mathrm{~h}$. Images were acquired with the Leica DMI6000 inverted microscope using a $20 \times$ magnification.

Clinical patient information All samples in this study was collected in Minhang Hospital College Hospital from 2019 to 2020. The patients of RA were diagnosed according to the 2010 ACR/EULAR classification criteria. This study was approved by the Medical Ethical Committee of Minhang Hospital affiliated Fudan university and fulfilled the ethical guidelines. Written informed consent was obtained from all participants.

Statistical analysis Statistical analyses were conducting using GraphPad Prism software (San Diego, CA, USA). Statistical analysis of differences between two groups was done using the independent-samples ttest and one-way ANOVA with Bonferroni's correction applied was used for comparison of more than two groups. Pearson's correlation coefficient was used for correlation analyses. $P<0.05$ was considered significant in all analyses.

\section{Results}

\section{GSDMD is activated in neutrophils from RA patients}

To investigate whether GSDMD contributes to the pathogenesis in RA disease, we first tested the level of its expression in neutrophils in peripheral blood of RA patients.

Flow cytometry analysis revealed increased cell population of $\mathrm{CD} 66^{+}$and GSDMD positive staining from peripheral blood of RA patients compared with healthy volunteer (HV). The median fluorescence intensity (MFI) of GSDMD was also significantly increased in neutrophils from peripheral blood of RA patients compared with HV (Figure 1, A and B). We next examined the cleaved pore-forming GSDMD-N fragment in neutrophils isolated from peripheral blood in RA patients. The expression level of full-length GSDMD was decreased and GSDMD-N was only detectable from RA patients (Figure 1, C and D). As GSDMDdependent pyroptosis is crucial for inflammatory cytokines secretion. We next investigated the level of IL$1 \beta$ and IL-18 in RA serum, and the level of IL-1 $\beta$ and IL-18 were both positively correlated with GSDMD-N expression (Figure 1, E and F). One of the most important roles of GSDMD is forming membrane pores through binding to plasma membrane. Immunofluorescent staining showed GSDMD was diffusely 
expressed in cytosolic region in normal human neutrophils but translocated to the plasma membrane (as indicated by the membrane protein Ly6G) in neutrophils from RA patients (Figure $1 \mathrm{G}$ ). Taken together, these experiments demonstrated that GSDMD was significantly activated in neutrophils from RA patients.

\section{GSDMD is required for NETs release after serum from RA patient treatment.}

We next examined whether GSDMD is required in mediating DNA release in RA. Neutrophils were isolated from HV, and cultured with serum from RA patients. Sytox green is a pivotal dye for detecting NETs [19]. An excessive NETs (as indicated by extracellular sytox green positively staining region) formation was observed in neutrophils after human RA serum. Disulfiram (DSF) was recently identified as an effective inhibitor of GSDMD [20]. And the release of extracellular DNA was significantly suppressed after DSF treatment (Figure 2, A and B). We next investigate the role of GSDMD in NETs formation in bone marrow neutrophils from mice. Since Immunofluorescent staining revealed NETs release (as indicated by cit-H3 and Sytox green) from wild-type neutrophils after serum treatment from CIA mice model. The release of NETs was significantly suppressed in Gsdmd'/neutrophils or DSF treatment (Figure 2, C and D). The results showed that GSDMD promotes NETs after RA serum treatment.

\section{Pyroptotic neutrophils promotes fibroblast-like synoviocytes (FLS) cells activation and proliferation}

Myeloid cells-induced Inflammation and activated hyperplastic fibroblast-like synoviocytes (FLS) play a key pathogenic role in rheumatoid arthritis (RA). We then investigate the interaction between these two subsets of cells. FLS were isolated from human synovial tissue from RA patents. There was no activation of FLS after RA serum treatment. However, when the cultured medium of FLS was replaced by neutrophil cultured medium after RA treatment (Figure 3A). Western blot showed the level of fibronectin (FN), Collagen-I (Col-I) and a-SMA were significantly increased after neutrophils culture medium treatment. And FLS activation was suppressed after DSF or PAD4 inhibitor treatment (Figure 3, B and C). The intensity of a-SMA was significantly increased after neutrophil culture medium treatment. And the intensity of a-SMA was suppressed after DSF or GSK484 treatment (Figure 3D). Proliferation of FLS was also increased after neutrophil culture medium treatment. The increased level of BrdU was suppressed after DSF or PAD4 inhibitor treatment (Figure 3, E). Collectively, these results indicated that GSDMD-dependent neutrophil pyroptosis promotes FLS activation and proliferation.

\section{GSDMD is activated in CIA-induced RA mouse model}

We next explore the expression of GSDMD in RA mouse model. Western blot results showed the protein level of GSDMD-N in joint homogenates was significantly increased in CIA mouse model, and the highest expression of GSDMD-N was at 8 weeks (Figure 4, A and B). Serum from CIA mice in 8 weeks significantly promotes the cleavage of GSDMD (Figure 4, C and D). Since caspase-1 and caspase-11 are crucial for GSDMD activation. We then detected the activation of caspase- 1 and caspase-11. The activation of caspase- 1 and caspase- 11 were significantly increased after RA serum treatment. Meanwhile, citrullinated histone $\mathrm{H} 3$ was also significantly increased after RA treatment, which indicated NETs formation. Immunofluorescent staining showed the translocation of GSDMD after CIA serum 
treatment. However, membrane translocation of GSDMD was significantly suppressed by caspase-1 and caspase-11 deficiency. Thus, these results suggest that GSDMD was activation in CIA-induced RA mouse model.

\section{Inhibition of GSDMD prevented arthritis progression and alleviated synovial inflammation in CIA mice}

To further detect the potential therapeutic effects of GSDMD on RA in vivo, CIA-induced RA mice model was used in the current study. A group of mice was administrated with $20 \mathrm{mg} / \mathrm{kg}$ of DSF three times a week after type II collagen boosting immunization (Figure 5A). The other group was only injected with the vehicle. The results showed that DSF treatment in CIA mice ameliorated arthritis incidence compared with vehicle-treated mice (Figure 5B). Also, DSF significantly attenuated arthritis severity in CIA animals, as demonstrated by alleviative erythema or swelling. The arthritis score in CIA was reduced by $30 \%$ at about 50 days after immunization, such that the peak score only reached a maximum of 2.5 , compared to the maximum of 6 reached by vehicle-treated CIA mice (Figure 5C). Pro-inflammatory cytokines play a key role in the pathogenesis of RA. Thus, pro-inflammatory cytokines in joint tissues were further determined. DSF significantly decreased the expression of IL-1 $\beta$, IL-6, IL-8 and TNF- $\alpha$ in CIA mice as detected by both mRNA and protein levels (Figure 5D). Collagenases and stromelysin are important for degradation of cartilage in RA, and activated FLS secret large amount of these proteins. We next investigate the expression of MMP1 and MMP3 in CIA mice. DSF significantly decreased the expression of MMP1 and MMP3 in CIA mice as detected by both mRNA and protein levels (Figure 5E). Taken together, these results showed that GSDMD promotes arthritis progression and alleviated synovial inflammation in CIA mice.

\section{Proinflammatory cytokines promotes FLS activation and proliferation}

Proinflammatory cytokines play an essential role in RA [21]. We next investigate whether these GSDMDdependent releasing proinflammatory cytokines facilitate FLS activation and proliferation. Recombinant IL-1 $\beta$ and TNF- $\alpha$ both promote the increase of fibronectin, Collagen-I and a-SMA expression in FLS (Figure $6, A$ and B). The level of BrdU was significantly increased after IL-1 $\beta$ and TNF- $\alpha$ treatment (Figure 6C). Collectively, the results showed that GSDMD-dependent IL-1 $\beta$ and TNF-a release promotes FLS activation and proliferation.

\section{Discussion}

NETs are extracellular fibers primarily composed of nucleic acids bound to neutrophil granule-derived proteins. The process of NETs formation has been associated with RA and increase of NETs has observed in RA $[22,23]$. However, whether NETs can be a therapeutic target in RA remains undetermined. Here, we first provide evidence that GSDMD mediated NETs formation contribute to the pathogenesis in RA. GSDMD inhibitor significantly reduced CIA-induced arthritis severity and loss of cartilage. These data suggested that GSDMD mediated NETs formation plays an important role in RA.

One of the most interesting results in our study is no activation of FLS after RA serum treatment. However, when we treated neutrophils with RA serum for $12 \mathrm{~h}$, and then added neutrophil culture medium 
into FLS. We observed significantly activation of FLS, as detected by more ECM protein synthesis and cell proliferation. Furthermore, the activation of FLS was suppressed by DSF or PAD4 inhibitor, which indicated that NETs plays an essential role in FLS activation. The contact between myeloid cells and activated hyperplastic FLS play a key pathogenic role in RA [5]. Previous work suggested NETs are an important source of citrullinated autoantigens in RA. Our results further indicated that NETs are able to activate a proinflammatory and pathogenic phenotype in FLS, and this process can be suppressed by GSDMD inhibitor. Meanwhile, a recent study showed that FLS from patients with RA have the capacity to internalize NETs, and present arthritogenic peptides to T cells, leading autoimmunity and cartilage damage $[24,25]$. So the interaction between neutrophils and FLS are interesting and much more sophisticated, Further studies are needed to explore this issue.

GSDMD, a newly identified pyroptosis executioner, has been reported to play an essential role in autoimmune disease $[13,14]$. Our results showed that the cleaved N-terminal of GSDMD was detected in RA patients and CIA-induced mouse model. In recent years, the expression and activation of GSDMD have been reported in neutrophils [26], and GSDMD-dependent pore formation acts as a conduit for IL-1 $\beta$ secretion [27]. GSDMD is widely expressed in different tissues and cell types, with high levels of expression in the macrophages and gastrointestinal epithelia [28]. Our results showed GSDMD was highly expressed in neutrophils as indicated by western blot or immunofluorescence. Furthermore, in vitro study, the cleaved GSDMD-N was significantly increased in neutrophils after RA serum treatment. Out results also showed the activation of caspase- 1 and caspase- 11 in neutrophils after CIA serum treatment, which indicated both caspase- 1 and caspase-11 promotes the cleavage of GSDMD. However, the precise mechanism about caspase- 1 and caspase-11 activation after RA serum treatment need further investigation.

It is well reported that CIA is one of common models of RA, and CIA has been widely applied in various kinds of studies to explore RA pathogenesis and discovery therapeutic targets for RA [29, 30]. Treatments of arthritic animals with DSF significantly suppressed proinflammatory cytokines (IL-1 $\beta$, IL-6, IL-8 and TNF-a), MMP1 and MMP 3 production in joint tissue. Pro-inflammatory cytokines, especially IL-1 $\beta$ and TNF-a potentiates the effects of growth factors (platelet-derived growth factor (PDGF) and transforming growth factor (TGF- $\beta$ ) on matrix metalloproteinase production and proliferation in FLS [31]. IL-1 $\beta$ has also been shown to reduce expression of DNA methylation enzymes in FLS [32]. MMP1 and MMP-3 are two important gelatinases in the MMP family. These two MMPs could not only efficiently cleave unfolded collagen but also cleave other matrix [33,34]. MMPs can activated by pro-inflammatory cytokines and have been found to be downregulated to anti-TNF therapy [35]. So GSDMD promote the pathogenesis of RA through regulating the secretion of pro-inflammatory cytokines and MMPs.

\section{Conclusions}

Our observations highlight a novel mechanism that promotes GSDMD-dependent immune dysregulation in RA, and further supports the rationale for testing NETs inhibitors and strategies that disrupt specific 
cell-cell interactions in the synovial joint in future clinical trials in RA. GSDMD may be a potential therapeutic target in RA.

\section{Declarations}

\section{Ethics approval and consent to participate}

All samples in this study was collected in Minhang Hospital College Hospital from 2019 to 2020 . The patients of RA were diagnosed according to the 2010 ACR/EULAR classification criteria. This study was approved by the Medical Ethical Committee of Minhang Hospital affiliated Fudan university and fulfilled the ethical guidelines. Written informed consent was obtained from all participants.

\section{Consent for publication}

All the authors are consent for publication.

\section{Availability of data and materials}

All the data generated or analyzed during this study are included in this article.

\section{Disclosure}

All the authors declared no competing interests.

\section{Funding}

This work is supported by the National Natural Science Foundation of China (Grant Number 81772433).

\section{Author contribution}

All authors were involved in drafting the article or revising it for important intellectual content, and all authors approved the final version to be published. K.Z. and C.B. designed and performed experiments, analyzed data and interpreted results. H.G., X.C., Z.H., G.C. helped with the experiments. X.Y and J.X. supervised the study and wrote the manuscript.

\section{Acknowledgements}

We would like to acknowledge the support of Prof. Lu for providing us with the methods about isolation FLS.

\section{References}

[1] J.S. Smolen, D. Aletaha, I.B. Mclnnes, Rheumatoid arthritis, Lancet, 388 (2016) 2023-2038. 
[2] G.S. Firestein, I.B. McInnes, Immunopathogenesis of Rheumatoid Arthritis, Immunity, 46 (2017) 183196.

[3] E. Choy, Understanding the dynamics: pathways involved in the pathogenesis of rheumatoid arthritis, Rheumatology, 51 Suppl 5 (2012) v3-11.

[4] H.L. Wright, R.J. Moots, S.W. Edwards, The multifactorial role of neutrophils in rheumatoid arthritis, Nature reviews. Rheumatology, 10 (2014) 593-601.

[5] R. Khandpur, C. Carmona-Rivera, A. Vivekanandan-Giri, A. Gizinski, S. Yalavarthi, J.S. Knight, S. Friday, S. Li, R.M. Patel, V. Subramanian, P. Thompson, P. Chen, D.A. Fox, S. Pennathur, M.J. Kaplan, NETs are a source of citrullinated autoantigens and stimulate inflammatory responses in rheumatoid arthritis, Science translational medicine, 5 (2013) 178ra140.

[6] C. Sur Chowdhury, S. Giaglis, U.A. Walker, A. Buser, S. Hahn, P. Hasler, Enhanced neutrophil extracellular trap generation in rheumatoid arthritis: analysis of underlying signal transduction pathways and potential diagnostic utility, Arthritis research \& therapy, 16 (2014) R122.

[7] K.W. Chen, M. Monteleone, D. Boucher, G. Sollberger, D. Ramnath, N.D. Condon, J.B. von Pein, P. Broz, M.J. Sweet, K. Schroder, Noncanonical inflammasome signaling elicits gasdermin D-dependent neutrophil extracellular traps, Science immunology, 3 (2018).

[8] G. Sollberger, A. Choidas, G.L. Burn, P. Habenberger, R. Di Lucrezia, S. Kordes, S. Menninger, J. Eickhoff, P. Nussbaumer, B. Klebl, R. Kruger, A. Herzig, A. Zychlinsky, Gasdermin D plays a vital role in the generation of neutrophil extracellular traps, Science immunology, 3 (2018).

[9] N. Kayagaki, I.B. Stowe, B.L. Lee, K. O'Rourke, K. Anderson, S. Warming, T. Cuellar, B. Haley, M. RooseGirma, Q.T. Phung, P.S. Liu, J.R. Lill, H. Li, J. Wu, S. Kummerfeld, J. Zhang, W.P. Lee, S.J. Snipas, G.S. Salvesen, L.X. Morris, L. Fitzgerald, Y. Zhang, E.M. Bertram, C.C. Goodnow, V.M. Dixit, Caspase-11 cleaves gasdermin D for non-canonical inflammasome signalling, Nature, 526 (2015) 666-671.

[10] J. Shi, Y. Zhao, K. Wang, X. Shi, Y. Wang, H. Huang, Y. Zhuang, T. Cai, F. Wang, F. Shao, Cleavage of GSDMD by inflammatory caspases determines pyroptotic cell death, Nature, 526 (2015) 660-665.

[11] J. Ding, K. Wang, W. Liu, Y. She, Q. Sun, J. Shi, H. Sun, D.C. Wang, F. Shao, Pore-forming activity and structural autoinhibition of the gasdermin family, Nature, 535 (2016) 111-116.

[12] X. Liu, Z. Zhang, J. Ruan, Y. Pan, V.G. Magupalli, H. Wu, J. Lieberman, Inflammasome-activated gasdermin $D$ causes pyroptosis by forming membrane pores, Nature, 535 (2016) 153-158.

[13] A. Kanneganti, R.K.S. Malireddi, P.H.V. Saavedra, L. Vande Walle, H. Van Gorp, H. Kambara, H. Tillman, P. Vogel, H.R. Luo, R.J. Xavier, H. Chi, M. Lamkanfi, GSDMD is critical for autoinflammatory pathology in a mouse model of Familial Mediterranean Fever, The Journal of experimental medicine, 215 (2018) 15191529. 
[14] S. Li, Y. Wu, D. Yang, C. Wu, C. Ma, X. Liu, P.N. Moynagh, B. Wang, G. Hu, S. Yang, Gasdermin D in peripheral myeloid cells drives neuroinflammation in experimental autoimmune encephalomyelitis, The Journal of experimental medicine, 216 (2019) 2562-2581.

[15] M.F. Bustamante, R. Garcia-Carbonell, K.D. Whisenant, M. Guma, Fibroblast-like synoviocyte metabolism in the pathogenesis of rheumatoid arthritis, Arthritis research \& therapy, 19 (2017) 110.

[16] T. Montero-Melendez, A. Nagano, C. Chelala, A. Filer, C.D. Buckley, M. Perretti, Therapeutic senescence via GPCR activation in synovial fibroblasts facilitates resolution of arthritis, Nature communications, 11 (2020) 745.

[17] L.J. O'Neil, M.J. Kaplan, Neutrophils in Rheumatoid Arthritis: Breaking Immune Tolerance and Fueling Disease, Trends in molecular medicine, 25 (2019) 215-227.

[18] E. Aga, D.M. Katschinski, G. van Zandbergen, H. Laufs, B. Hansen, K. Muller, W. Solbach, T. Laskay, Inhibition of the spontaneous apoptosis of neutrophil granulocytes by the intracellular parasite Leishmania major, Journal of immunology, 169 (2002) 898-905.

[19] S.K. Jorch, P. Kubes, An emerging role for neutrophil extracellular traps in noninfectious disease, Nature medicine, 23 (2017) 279-287.

[20] J.J. Hu, X. Liu, S. Xia, Z. Zhang, Y. Zhang, J. Zhao, J. Ruan, X. Luo, X. Lou, Y. Bai, J. Wang, L.R. Hollingsworth, V.G. Magupalli, L. Zhao, H.R. Luo, J. Kim, J. Lieberman, H. Wu, FDA-approved disulfiram inhibits pyroptosis by blocking gasdermin D pore formation, Nature immunology, 21 (2020) 736-745.

[21] M. Noack, P. Miossec, Selected cytokine pathways in rheumatoid arthritis, Seminars in immunopathology, 39 (2017) 365-383.

[22] E. Neumann, R. Hasseli, U. Lange, U. Muller-Ladner, The Role of Extracellular Nucleic Acids in Rheumatoid Arthritis, Current pharmaceutical biotechnology, 19 (2018) 1182-1188.

[23] F. Apel, A. Zychlinsky, E.F. Kenny, The role of neutrophil extracellular traps in rheumatic diseases, Nature reviews. Rheumatology, 14 (2018) 467-475.

[24] D. Ummarino, Rheumatoid arthritis: Don't swallow the NETs, Nature reviews. Rheumatology, 13 (2017) 322.

[25] C. Carmona-Rivera, P.M. Carlucci, E. Moore, N. Lingampalli, H. Uchtenhagen, E. James, Y. Liu, K.L. Bicker, H. Wahamaa, V. Hoffmann, A.I. Catrina, P. Thompson, J.H. Buckner, W.H. Robinson, D.A. Fox, M.J. Kaplan, Synovial fibroblast-neutrophil interactions promote pathogenic adaptive immunity in rheumatoid arthritis, Science immunology, 2 (2017).

[26] R. Heilig, M.S. Dick, L. Sborgi, E. Meunier, S. Hiller, P. Broz, The Gasdermin-D pore acts as a conduit for IL-1beta secretion in mice, European journal of immunology, 48 (2018) 584-592. 
[27] J. Shi, W. Gao, F. Shao, Pyroptosis: Gasdermin-Mediated Programmed Necrotic Cell Death, Trends in biochemical sciences, 42 (2017) 245-254.

[28] N. Saeki, Y. Kuwahara, H. Sasaki, H. Satoh, T. Shiroishi, Gasdermin (Gsdm) localizing to mouse Chromosome 11 is predominantly expressed in upper gastrointestinal tract but significantly suppressed in human gastric cancer cells, Mammalian genome : official journal of the International Mammalian Genome Society, 11 (2000) 718-724.

[29] D.D. Brand, K.A. Latham, E.F. Rosloniec, Collagen-induced arthritis, Nature protocols, 2 (2007) 12691275 .

[30] M. Miyoshi, S. Liu, Collagen-Induced Arthritis Models, Methods in molecular biology, 1868 (2018) 3-7.

[31] S. Rosengren, M. Corr, D.L. Boyle, Platelet-derived growth factor and transforming growth factor beta synergistically potentiate inflammatory mediator synthesis by fibroblast-like synoviocytes, Arthritis research \& therapy, 12 (2010) R65.

[32] K. Nakano, D.L. Boyle, G.S. Firestein, Regulation of DNA methylation in rheumatoid arthritis synoviocytes, Journal of immunology, 190 (2013) 1297-1303.

[33] S. Genevay, A. Finckh, F. Mezin, E. Tessitore, P.A. Guerne, Influence of cytokine inhibitors on concentration and activity of MMP-1 and MMP-3 in disc herniation, Arthritis research \& therapy, 11 (2009) R169.

[34] P.S. Burrage, K.S. Mix, C.E. Brinckerhoff, Matrix metalloproteinases: role in arthritis, Frontiers in bioscience : a journal and virtual library, 11 (2006) 529-543.

[35] V.J. Richardson, Divergent and synergistic regulation of matrix metalloprotease production by cytokines in combination with C-C chemokines, International journal of immunopathology and pharmacology, 23 (2010) 715-726.

\section{Figures}


A

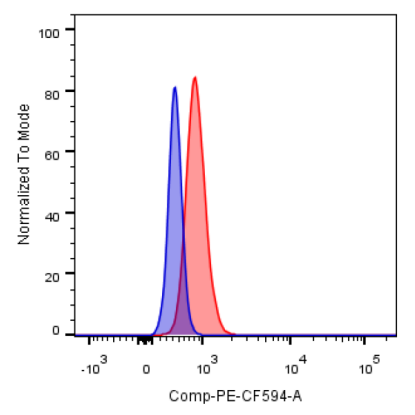

C

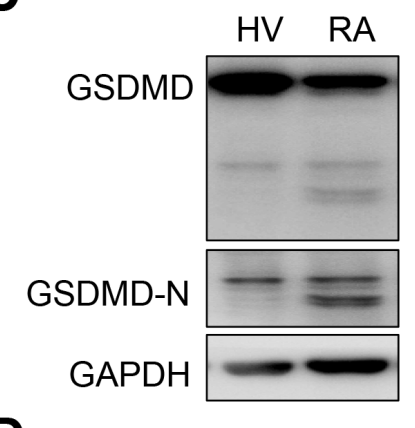

D

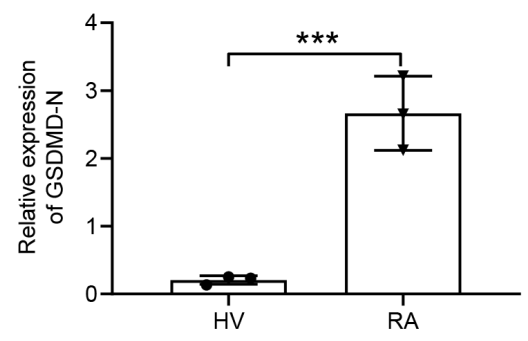

B

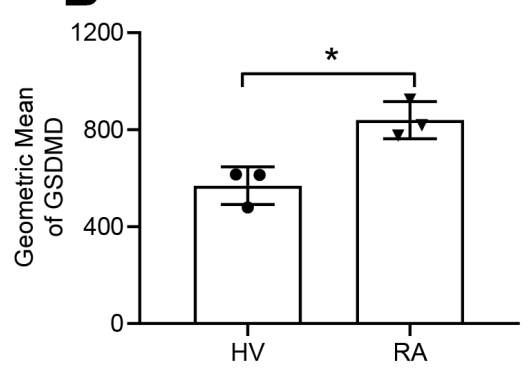

E

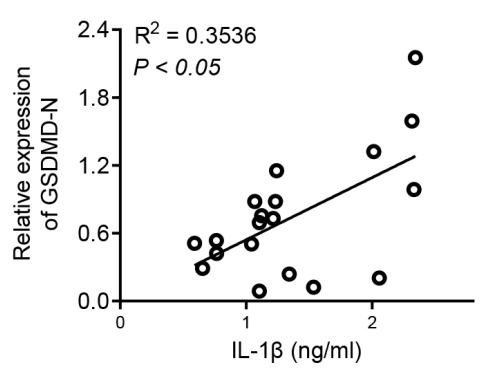

G HV

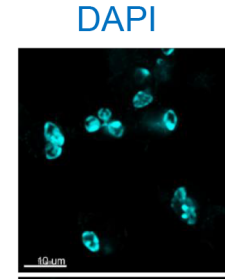

Ly6G

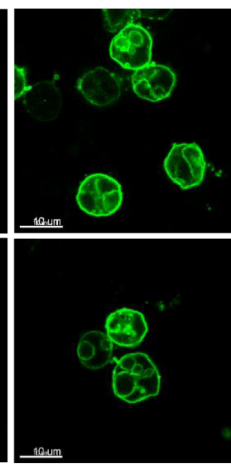

$\mathbf{F}$

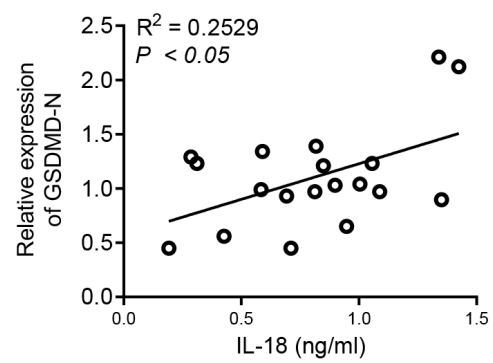

GSDMD

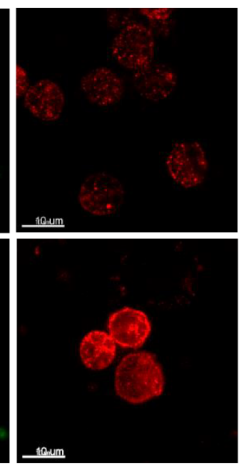

Merge

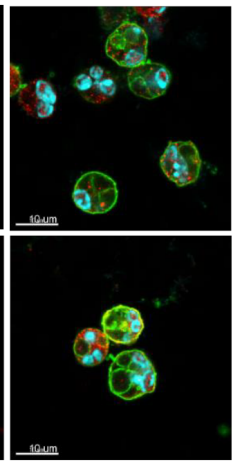

\section{Figure 1}

GSDMD is activated in neutrophils from RA patients. (A) Flow cytometric analysis of GSDMD and CD66 from peripheral blood of healthy volunteer (HV) and RA patients. (B) Median fluorescence intensity (MFI) and quantitative analysis of GSDMD in HV and RA patients. Data are presented as the mean \pm SEM $(n=$ 3). ${ }^{*} P<0.05$. (C) Immunoblot analysis of GSDMD and GSDMD-N terminal in neutrophils from peripheral blood of HV and RA patients. (D) Quantitative analysis of GSDMD-N in D. $* \star * P<0.001$. (E) Analysis of linear correlation between GSDMD-N level and IL-1 $\beta$ in RA ( $R 2=0.3513, P<0.05, n=19)$. ( $F$ ) Analysis of linear correlation between GSDMD-N level and IL-18 in RA (R2 = 0.357, $P<0.05, n=19)$. (G) Immunofluorescent staining analysis of neutrophils from HV and RA patients. Scale bar $=10 \mu \mathrm{m}$. 

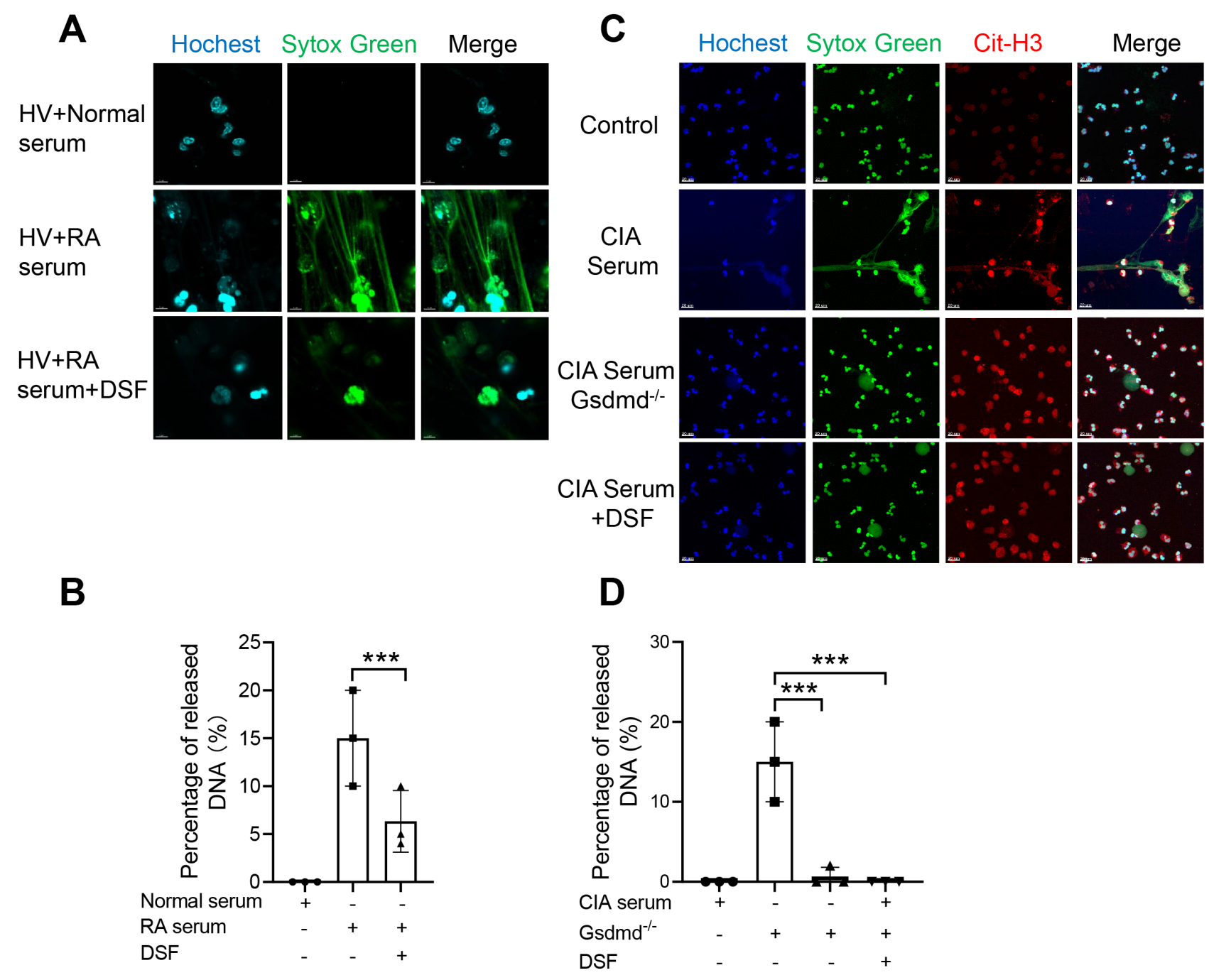

Figure 2

GSDMD is required for NETs release after RA serum treatment. (A) Immunofluorescent staining analysis of NETs in bone marrow neutrophils treated with HV+Normal serum, HV+RA serum, or HV+RA serum+DSF for $12 \mathrm{~h}$. Scale bar $=5 \mu \mathrm{m}$. (B) Quantitative analysis of NETs in A. Data are presented as the mean \pm SEM $(n=3)$. ${ }^{* \star} P<0.001$. (C) Immunofluorescent staining analysis of NETs in WT or Gsdmd-/- bone marrow neutrophils after CIA mouse treatment. For DSF group, WT bone marrow neutrophils were pretreated with DSF $(5 \mu \mathrm{M})$ for $2 \mathrm{~h}$. Scale bar $=20 \mu \mathrm{m}$. (D) Quantitative analysis of NETs in C. Data are presented as the mean $\pm \operatorname{SEM}(n=3)$. ***P $<0.001$. 
A

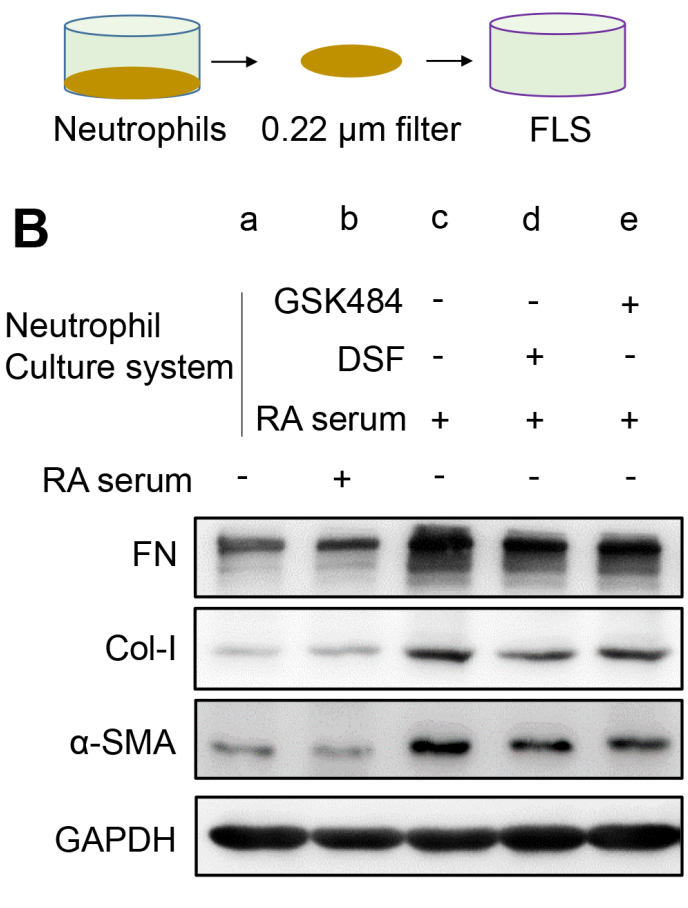

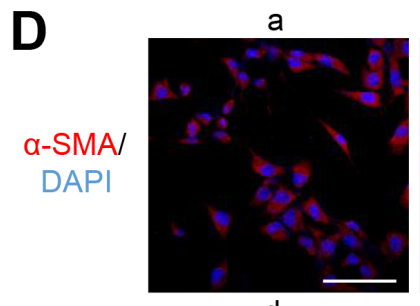

d
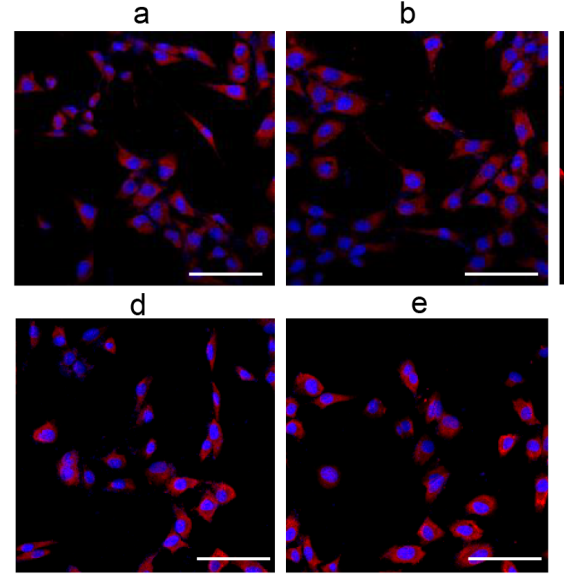

e

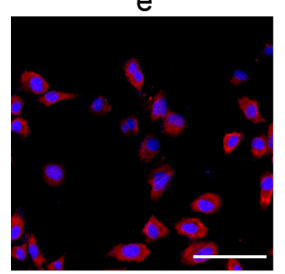

$\mathbf{E}$

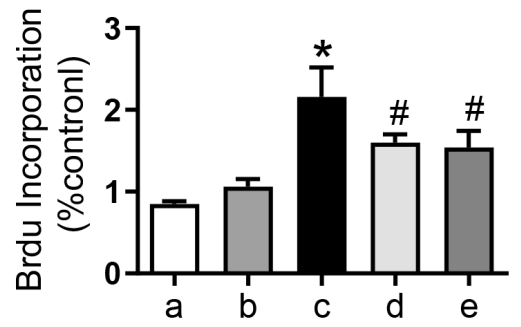

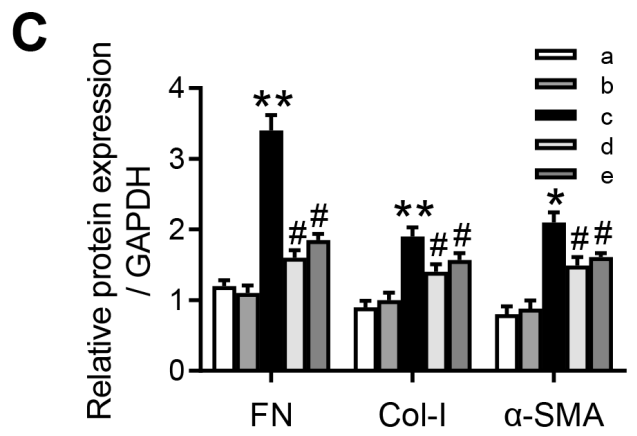

\section{Figure 3}

Pyroptotic neutrophils promotes fibroblast-like synoviocyte (FLS) activation and proliferation. (A) Diagram of FLS stimulated with culture medium from neutrophils after RA serum treatment. (B) Neutrophils were isolated from peripheral blood of HV. Synoviocytes were isolated by enzymatic digestion of synovial tissue specimens. Neutrophils were treated with RA serum (10\%) for $12 \mathrm{~h}$ (c: FLS were treated with RA serum, d: FLS were treated with DSF and RA serum, e: FLS were pretreated with GSK484 and RA serum). Then the cultured medium was filtered, and then totally added to culture FLS (a: control FLS, b: FLS with RA serum treatment). Immunoblot analysis of fibronectin (FN), Collagen-I (Col-I) and a-SMA. (C) Quantitative analysis of the indicated proteins in B. Data are presented as the mean \pm SEM $(n=3)$. ${ }^{P}<$ $0.05,{ }^{*} \mathrm{P}<0.01$ compared with $\mathrm{a}, \# \mathrm{P}<0.05$ compared with $\mathrm{c}$. (D) Confocal analysis of a-SMA staining of FLS. Scale bar $=30 \mu \mathrm{m}$. (E) ELISA of Brdu detection in FLS. Data are presented as the mean \pm SEM $(n=$ 3). ${ }^{*} \mathrm{P}<0.05$ compared with $\mathrm{a}, \# \mathrm{P}<0.05$ compared with $\mathrm{C}$. 

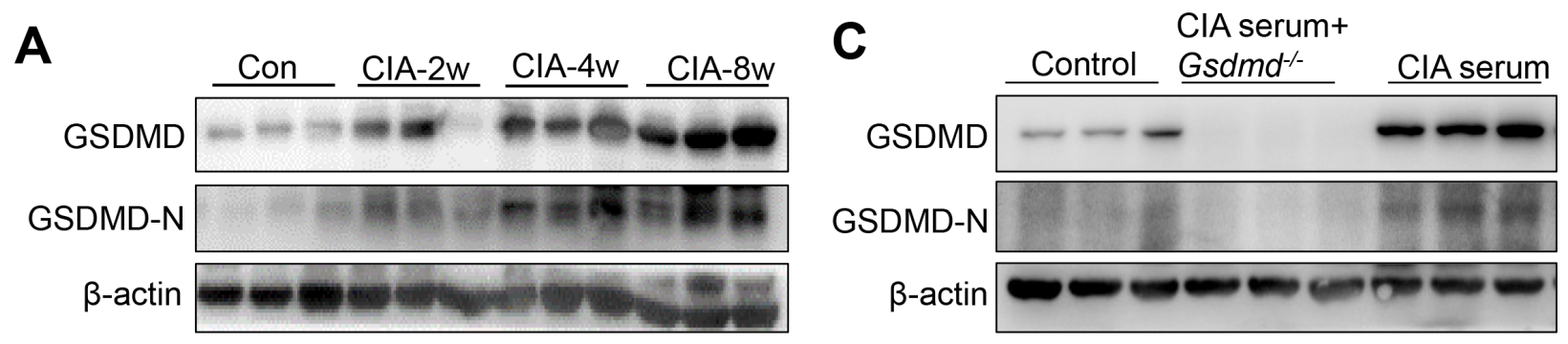

B

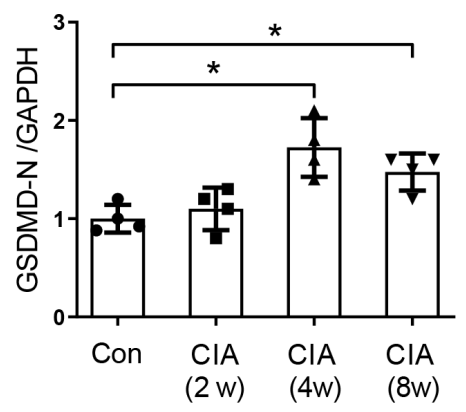

D
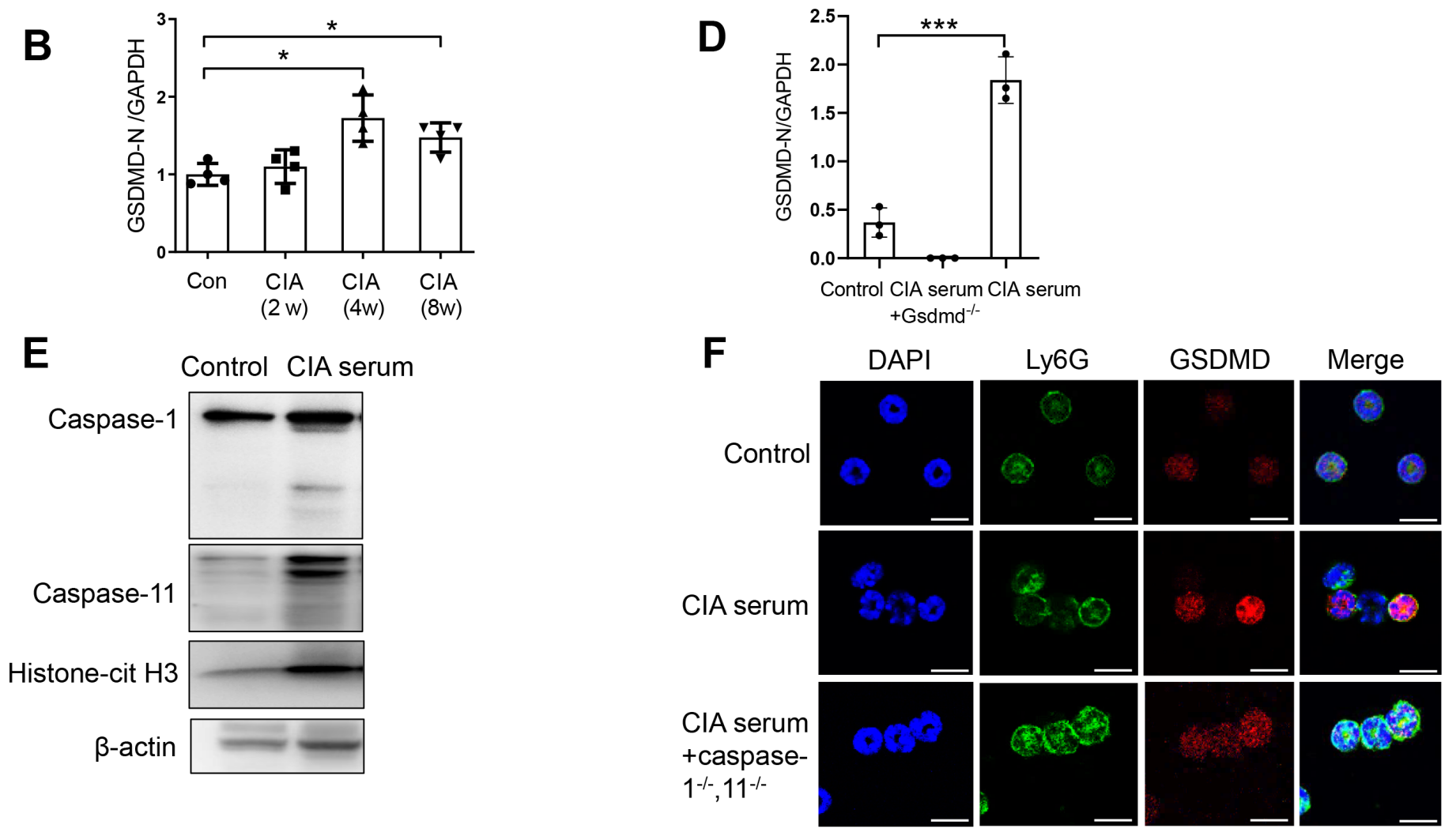

Figure 4

GSDMD is activated in CIA-induced RA mouse model. (A) Immunoblot analysis of GSDMD and GSDMD-N in joint homogenates isolated of mice from Con, $\mathrm{CIA} 2 \mathrm{w}, \mathrm{CIA} 4 \mathrm{w}$ and $\mathrm{CIA} 8 \mathrm{w}$. (B) Quantitative analysis of GSDMD-N in A. Data are presented as the mean \pm SEM $(n=3)$. ${ }^{*}<0.05$. (C) Immunoblot analysis of GSDMD and GSDMD-N in WT or Gsdmd-/- bone marrow neutrophils after CIA serum treatment. (D) Quantitative analysis of GSDMD-N in C. Data are presented as the mean \pm SEM $(n=3)$. ${ }^{\star \star \star} P<0.001$. (E) Immunoblot analysis of caspase-1, caspase-11 and Histone-cit H3 in WT bone marrow neutrophils treated with control or RA serum. (F) Immunofluorescent staining analysis of GSDMD in WT bone marrow neutrophils treated with Con, CIA serum and CIA serum+DSF. Scale bar $=15 \mu \mathrm{m}$. 
A

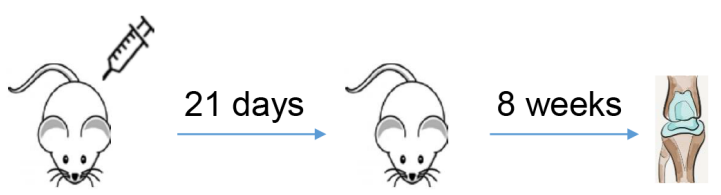

Type II Collagen

DSF $(20 \mathrm{mg} / \mathrm{kg})$ three times a week

Harvest joint

C

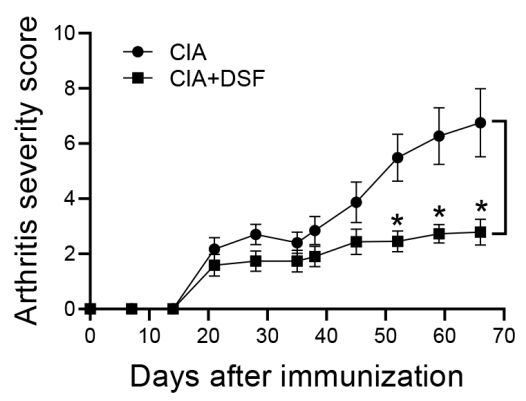

E

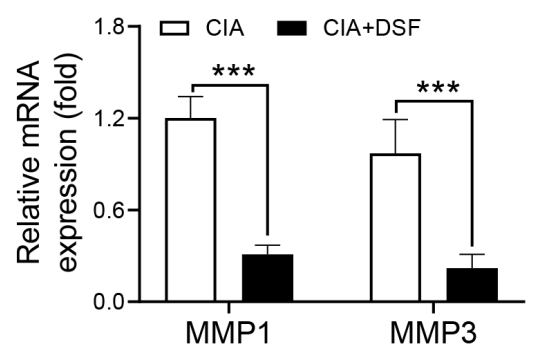

B

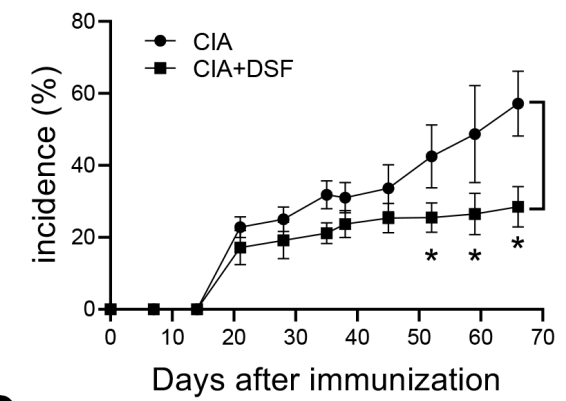

D

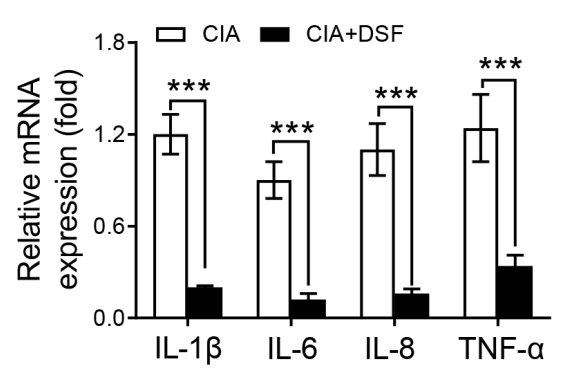

F

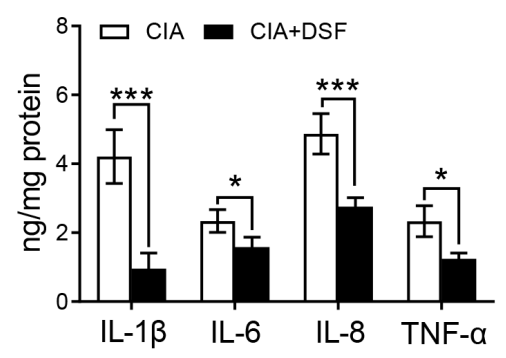

\section{Figure 5}

Inhibition of GSDMD prevented arthritis progression and alleviated synovial inflammation in CIA mice. (A) Diagram of the RA mice model conduction. (B) Incidence in CIA and CIA+DSF. ${ }^{*}<0.05$. (C) arthritis severity in CIA and CIA+DSF. *P $<0.05$. (D) The mRNA level of IL-1 $\beta$, IL-6, IL-8 and TNF- $\alpha$ in joint of CIA mice and CIA+DSF mice. ${ }^{*} \times P<0.001$. (E) The mRNA level of MMP1 and MMP3 in joint of CIA mice and CIA+DSF mice. ${ }^{* * *} P<0.001$. (F) ELISA detection of IL-1 $\beta$, IL-6, IL-8 and TNF-a in joint of CIA mice and CIA+DSF mice. ${ }^{*} \mathrm{P}<0.05 .{ }^{* *} \mathrm{P}<0.001$. 
A

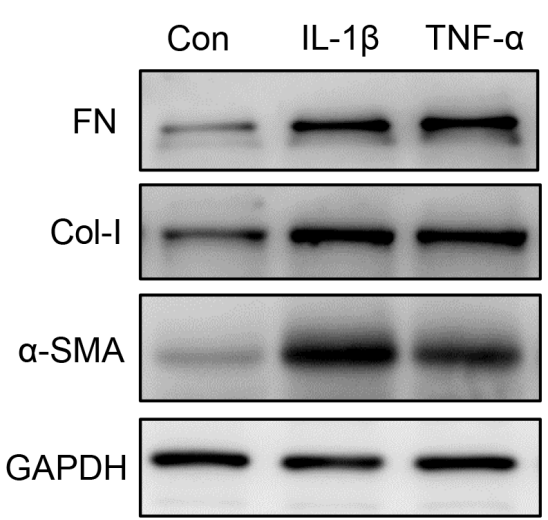

B

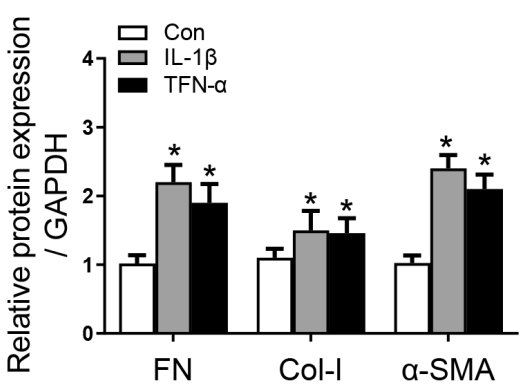

C

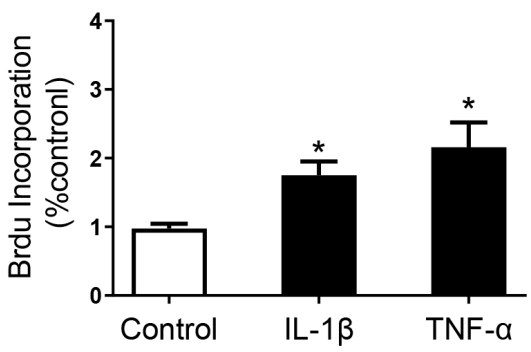

\section{Figure 6}

IL-1 $\beta$ and TNF- $\alpha$ promotes FLS activation and proliferation. FLS was isolated from SLE patients. (A) Immunoblot analysis of fibronectin (FN), Collagen-I (Col-I) and a-SMA. (B) Quantitative analysis of fibronectin (FN), Collagen-I (Col-I) and a-SMA. Data are presented as the mean \pm SEM $(n=3)$. ${ }^{*} P<0.05$. (C) ELISA of Brdu detection in FLS. Data are presented as the mean \pm SEM $(n=3)$. $* P<0.05$ compared with Con. 